\title{
A Result regarding the Seismic Dislocations in Microstretch Thermoelastic Bodies
}

\author{
M. Marin, ${ }^{1}$ O. Florea, ${ }^{1}$ and S. R. Mahmoud ${ }^{2,3}$ \\ ${ }^{1}$ Department of Mathematics, Transilvania University of Brasov, 500091 Brasov, Romania \\ ${ }^{2}$ Department of Mathematics, King Abdulaziz University, Jeddah 21589, Saudi Arabia \\ ${ }^{3}$ Department of Mathematics, Sohag University, Sohag 82524, Egypt
}

Correspondence should be addressed to M. Marin; m.marin@unitbv.ro

Received 8 December 2014; Revised 16 January 2015; Accepted 20 January 2015

Academic Editor: Chunlin Chen

Copyright (C) 2015 M. Marin et al. This is an open access article distributed under the Creative Commons Attribution License, which permits unrestricted use, distribution, and reproduction in any medium, provided the original work is properly cited.

\begin{abstract}
The aim of our study is to derive a relation of De Hoop-Knopoff type for displacement fields within context of thermoelastic microstretch bodies. Then, as a consequence, an explicit expression of the body loadings equivalent to a seismic dislocation is obtained. The results are extensions of those from the classical theory of elastic bodies.
\end{abstract}

\section{Introduction}

The theory of thermomicrostretch elastic solids was first elaborated by Eringen [1], and, in short, this is a theory of thermoelasticity with microstructure that includes intrinsic rotations and microstructural expansion and contractions.

The purpose of this theory is to eliminate discrepancies between classical elasticity and experiments, since the classical elasticity failed to present acceptable results when the effects of material microstructure were known to contribute significantly to the body's overall deformations, for example, in the case of granular bodies with large molecules (e.g., polymers), graphite, or human bones.

These cases are becoming increasingly important in the design and manufacture of modern day advanced materials, as small-scale effects become paramount in the prediction of the overall mechanical behaviour of these materials.

Other intended applications of this theory are to composite materials reinforced with chopped fibers and various porous materials.

This theory can be useful in the applications which deal with porous materials as geological materials, solid packed granular materials, and many others.

On the other hand, materials which operate at elevated temperatures will invariably be subjected to heat flow at some time during normal use. Such heat flow will involve a nonlinear temperature distribution which will inevitably give rise to thermal stresses. For these reasons, the development, design, and selection of materials for high temperature applications require a great deal of care. The role of the pertinent material properties and other variables which can affect the magnitude of thermal stress must be considered.

The main difficulty of the thermomicrostretch materials is the large number of the thermoelastic coefficients and, as such, the problem of their determination in the laboratory. Yet many authors consider that this problem will be solved in the future.

Already, in the isotropic case, when the number of coefficients decreases a lot, they are calculated as can be seen in many works due to Eringen or Iesan.

The present paper must be considered a first step to a better understanding of microstretch and thermal stress in the study of above enumerated materials.

The reciprocity and representation relations that appear in our study constitute powerful theoretical tools in the assessment of the theory of seismic-sources mechanism, in the studies connected with seismic wave propagation.

Also, we think that this paper is a good help to understand the application of microstretch mechanism to earthquake problems.

There are many results regarding the mechanism of earthquake, as in [2-8]. 
For instance, in [6], the authors establish a reciprocity relation which forms the basis for a uniqueness result, a continuous dependence of solutions upon initial data and body loads and a variational characterization of solutions. The effect of a concentrated heat supply and of a concentrated heat volume charge density in an unbounded homogeneous and isotropic electromagnetic body is investigated.

We find other results regarding thermoelasticity of nonclassical materials, as in [1,9-12]. The main results of our study are extensions of some similar results in the classical elasticity in order to cover the thermoelasticity of microstretch bodies (see the results due to Saccomandi in [13]).

\section{Basic Equations}

For convenience the notations and terminology chosen are almost identical to those of our study [11]. Our paper is concerned with an anisotropic and homogeneous material.

Let the body occupy, at time $t=0$, a properly regular region $B$ of the three-dimensional Euclidian space, bounded by the piece-wise smooth surface $\partial B$ and we denote the closure of $B$ by $\bar{B}$. We refer the motion of the body to a fix system of rectangular Cartesian axes $O x_{i}, i=1,2,3$ and adopt the Cartesian tensor notation. Points in $B$ are denoted by $x_{j}$ and $t \in[0, \infty)$ is temporal variable. Throughout this work the Einstein summation convention over repeated indices is used. The subscript $j$ after comma indicates partial differentiation with respect to the spatial argument $x_{j}$. All Latin subscripts are understood to range over the integers $(1,2,3)$, while the Greek indices have the range $(1,2)$. A superposed dot denotes the derivatives with respect to the $t$-time variable. Also, the spatial argument and the time argument of a function will be omitted when there is no likelihood of confusion.

Let us denote by $u_{i}$ the components of the displacement vector and by $\varphi_{i}$ the components of the microrotation vector. Also, we denote by $\omega$ the microstretch function and by $\theta$ the temperature measured from the constant absolute temperature $T_{0}$ of the body in its reference state.

As usual, we denote by $t_{i j}$ the components of the stress tensor and by $m_{i j}$ the components of the couple stress tensor over $B$. Also, we denote by $\lambda_{i}$ the components of the microstress vector.

For clarity and simplicity in presentation, the regularity hypotheses on the considered functions will be ommited.

On these grounds, the field equations in the dynamic theory of thermoelasticity of microstretch bodies are as follows (see $[1,11,14])$ :

(i) the equations of motion

$$
\begin{gathered}
t_{j i, j}+\varrho F_{i}=\varrho \ddot{u}_{i}, \\
m_{j i, j}+\varepsilon_{i j k} t_{j k}+\varrho G_{i}=I_{i j} \ddot{\varphi}_{j} ;
\end{gathered}
$$

(ii) the balance of the equilibrated forces

$$
\lambda_{i, i}-s+\varrho L=J \ddot{\omega} .
$$

The equation of energy is given by

$$
\varrho T_{0} \dot{\eta}=q_{i, i}+\varrho r
$$

In the above equations we have used the following notations:

(i) $F_{i}$ are the components of body force;

(ii) $G_{i}$ are the components of body couple;

(iii) $L$ is the generalized external body load;

(iv) $s$ is the generalized internal body load;

(v) $\varrho$ is the reference constant mass density;

(vi) $J$ and $I_{i j}=I_{j i}$ are the coefficients of microinertia;

(vii) $\eta$ is the entropy per unit mass;

(viii) $r$ is the heat supply per unit mass;

(ix) $q_{i}$ are the components of heat flux vector.

For an anisotropic and homogeneous microstretch thermoelastic material, the constitutive equations have the form:

$$
\begin{gathered}
t_{i j}=A_{i j r s} \varepsilon_{r s}+B_{i j r s} \mu_{r s}+D_{i j r} \gamma_{r}+a_{i j} \omega-E_{i j} \theta, \\
m_{i j}=B_{r s i j} \varepsilon_{r s}+C_{i j r s} \mu_{r s}+E_{i j r} \gamma_{r}+b_{i j} \omega-D_{i j} \theta, \\
\lambda_{i}=D_{r s i} \varepsilon_{r s}+E_{r s i} \mu_{r s}+C_{i j} \gamma_{j}+d_{i} \omega-L_{i} \theta, \\
s=a_{i j} \varepsilon_{i j}+b_{i j} \mu_{i j}+d_{i} \gamma_{i}+m \omega-\alpha \theta, \\
\eta=\eta_{0}+E_{i j} \varepsilon_{i j}+D_{i j} \mu_{i j}+L_{i} \gamma_{i}+\alpha \omega+\frac{a}{T_{0}} \theta, \\
q_{i}=k_{i j} \theta_{, j},
\end{gathered}
$$

where $A_{i j r s}, B_{i j r s}, C_{i j r s}, D_{i j r}, E_{i j r}, a_{i j}, b_{i j}, c_{i j}, d_{i}, E_{i j}, D_{i j}, L_{i}, m$, $\alpha, a$, and $k_{i j}$ are the characteristic constitutive coefficients.

The components of the strain tensors $\varepsilon_{i j}, \mu_{i j}$, and $\gamma_{i}$ are defined by means of the geometric equations:

$$
\varepsilon_{i j}=u_{j, i}+\varepsilon_{i j k} \varphi_{k}, \quad \mu_{i j}=\varphi_{j, i}, \quad \gamma_{i}=\omega_{, i},
$$

where $\varepsilon_{i j k}$ is the alternating symbol.

The constitutive coefficients obey the following symmetry relations:

$$
\begin{gathered}
A_{i j r s}=A_{r s i j}, \quad C_{i j r s}=C_{r s i j}, \\
C_{i j}=C_{j i}, \quad k_{i j}=k_{j i} .
\end{gathered}
$$

One can assume that a positive constant $\lambda_{0}$ exists such that

$$
I_{i j} \xi_{i} \xi_{j} \geq \lambda_{0} \xi_{i} \xi_{i}, \quad \forall \xi_{i}
$$

Also, the Second Law of Thermodynamics implies that

$$
k_{i j} \xi_{i} \xi_{j} \geq 0, \quad \forall \xi_{i}
$$

We denote by $t_{i}$ the components of surface traction, $m_{i}$ the components of surface couple, $p$ the microsurface traction, and $q$ the heat flux. These quantities are defined by

$$
\begin{gathered}
t_{i}=t_{j i} n_{j}, \quad m_{i}=m_{j i} n_{j}, \\
p=\lambda_{i} n_{i}, \quad q=q_{i} n_{i},
\end{gathered}
$$

at regular points of the surface $\partial B$. 
Here, $n_{i}$ are the components of the outward unit normal of the surface $\partial B$.

Along with the system of field equations (1)-(5) we consider the following initial conditions:

$$
\begin{gathered}
u_{i}(x, 0)=u_{i}^{0}(x), \quad \dot{u}_{i}(x, 0)=u_{i}^{1}(x), \\
\varphi_{i}(x, 0)=\varphi_{i}^{0}(x), \quad \dot{\varphi}_{i}(x, 0)=\varphi_{i}^{1}(x), \\
\omega(x, 0)=\omega^{0}(x), \quad \dot{\omega}(x, 0)=\omega^{1}(x), \\
\theta(x, 0)=\theta^{0}(x), \\
x \in \bar{B}
\end{gathered}
$$

and the following prescribed boundary conditions:

$$
\begin{gathered}
u_{i}=\bar{u}_{i} \quad \text { on } \partial B_{1} \times\left[0, t_{0}\right), \\
t_{i}=\bar{t}_{i} \quad \text { on } \partial B_{1}^{c} \times\left[0, t_{0}\right), \\
\varphi_{i}=\bar{\varphi}_{i} \quad \text { on } \partial B_{2} \times\left[0, t_{0}\right), \\
m_{i}=\bar{m}_{i} \quad \text { on } \partial B_{2}^{c} \times\left[0, t_{0}\right), \\
\omega=\bar{\omega} \quad \text { on } \partial B_{3} \times\left[0, t_{0}\right), \\
p=\bar{p} \quad \text { on } \partial B_{3}^{c} \times\left[0, t_{0}\right), \\
\theta=\bar{\theta} \quad \text { on } \partial B_{4} \times\left[0, t_{0}\right), \\
q=\bar{q} \quad \text { on } \partial B_{4}^{c} \times\left[0, t_{0}\right),
\end{gathered}
$$

where $t_{0}$ is some instant that may be infinite.

Also, $\partial B_{1}, \partial B_{2}, \partial B_{3}$, and $\partial B_{4}$ with respective complements $\partial B_{1}^{c}, \partial B_{2}^{c}, \partial B_{3}^{c}$, and $\partial B_{4}^{c}$ are subsets of the surface $\partial B$ such that

$$
\begin{gathered}
\partial B_{1} \cap \partial B_{1}^{c}=\partial B_{2} \cap \partial B_{2}^{c}=\partial B_{3} \cap \partial B_{3}^{c}=\partial B_{4} \cap \partial B_{4}^{c}=\emptyset \\
\partial B_{1} \cup \partial B_{1}^{c}=\partial B_{2} \cup \partial B_{2}^{c}=\partial B_{3} \cup \partial B_{3}^{c}=\partial B_{4} \cup \partial B_{4}^{c}=\partial B
\end{gathered}
$$

Also, $u_{i}^{0}, u_{i}^{1}, \varphi_{i}^{0}, \varphi_{i}^{1}, \omega^{0}, \omega^{1}, \theta^{0}, \bar{u}_{i}, \bar{t}_{i}, \bar{\varphi}_{i}, \bar{m}_{i}, \bar{\omega}, \bar{p}, \bar{\theta}$, and $\bar{q}$ are prescribed functions in their domains.

By a solution of the mixed initial boundary value problem of the theory thermoelasticity of dipolar bodies with voids in the cylinder $\Omega_{0}=B \times\left[0, t_{0}\right)$ we mean an ordered array $\left(u_{i}\right.$, $\varphi_{i}, \omega$, and $\theta$ ) which satisfies (1), (2), and (3) for all $(x, t) \in \Omega_{0}$, the boundary conditions (11) and the initial conditions (10).

\section{Main Results}

Let $u$ and $v$ be functions defined on $\bar{B} \times[0, \infty)$ and continuous on $[0, \infty)$ with respect to the time variable $t$ for each spatial variable $x \in \bar{B}$.

We denote by $u * v$ the convolution of $u$ and $v$; that is,

$$
(u * v)(x, t)=\int_{0}^{t} u(x, t-\tau) v(x, \tau) d \tau .
$$

Let us introduce the notations

$$
\begin{gathered}
g(t)=t, \quad h(t)=1, \\
f_{i}=\varrho g * F_{i}+\varrho\left[t u_{i}^{1}(x)+u_{i}^{0}(x)\right], \\
g_{i}=\varrho g * G_{i}+I_{i j}\left[t \varphi_{j}^{1}(x)+\varphi_{j}^{0}(x)\right], \\
l=\varrho g * L+J\left[t \omega^{1}(x)+\omega^{0}(x)\right], \\
w=\varrho h * r+\varrho T_{0} \eta_{0} .
\end{gathered}
$$

Following the same procedure used by Ieşan in [5], it is easy to prove the following result that enables us to give an alternative formulation of the initial boundary value problem in which the initial data are incorporated into the field of equations.

Theorem 1. The functions $u_{i}, \varphi_{j k}, \sigma, \theta, \tau_{i j}, \eta_{i j}, \mu_{i j k}$, and $q_{i}$ satisfy (1), (2), (3), and the initial conditions (10) if and only if they satisfy the following system of equations:

$$
\begin{gathered}
g * t_{j i, j}+f_{i}=\varrho u_{i}, \\
g *\left(m_{j i, j}+\varepsilon_{i j k} t_{j k}\right)+g_{i}=I_{i j} \varphi_{j}, \\
g *\left(\lambda_{i, i}-s\right)+l=J \omega, \\
h * q_{i, i}+w=\varrho T_{0} \eta .
\end{gathered}
$$

In our following estimations, we will use formulation (15) of the mixed problem. We wish to find the behavior of the considered medium when embedded in $B$ there is a discontinuity surface $\Sigma$ for the displacements, the microration vector, the microstretch function, and the temperature. The sides of $\Sigma$ are denoted by $\Sigma^{-}$and $\Sigma^{+}$.

Let $v_{i}$ be the components of the unit normal vector of $\Sigma$, directed from the side $(-)$ to the side $(+)$.

Then on surface $\Sigma$ we have the conditions

$$
\begin{aligned}
u_{i}^{+}-u_{i}^{-}=U_{i}, & t_{j i}^{+} v_{j}=t_{j i}^{-} v_{j}, \\
\varphi_{i}^{+}-\varphi_{i}^{-}=\Phi_{i}, & m_{i j}^{+} v_{j}=m_{i j}^{-} v_{j} \\
\omega^{+}-\omega^{-}=\Psi, & \lambda_{j}^{+} v_{j}=\lambda_{j}^{-} v_{j}, \\
\theta^{+}-\theta^{-}=\Theta, & q_{j}^{+} v_{j}=q_{j}^{-} v_{j},
\end{aligned}
$$

where $f^{+}$and $f^{-}$are the limits of the function $f(x)$ as $x$ approaches a point on the side $(+)$ or $(-)$ of the surface $\Sigma$, respectively, and $U_{i}, \Phi_{i}, \Psi$, and $\Theta$ are prescribed functions. In this way we can consider (15) in the domain $B \backslash \Sigma$.

Let us consider two different systems of loadings for the body

$$
\begin{aligned}
\mathscr{G}^{(\alpha)}= & \left\{F_{i}^{(\alpha)}, G_{i}^{(\alpha)}, L^{(\alpha)}, r^{(\alpha)}, \bar{u}_{i}^{(\alpha)}, \bar{\varphi}_{i}^{(\alpha)}, \bar{\omega}_{i}^{(\alpha)}, \bar{\theta}^{(\alpha)},\right. \\
& \left.\bar{t}_{i}^{(\alpha)}, \bar{m}_{i}^{(\alpha)}, \bar{h}^{(\alpha)}, \bar{q}^{(\alpha)}, U_{i}, \Phi_{i}, \Psi, \Theta\right\}, \quad \alpha=1,2
\end{aligned}
$$


and two corresponding solutions

$$
\begin{gathered}
\mathcal{S}^{(\alpha)}=\left\{u_{i}^{(\alpha)}, \varphi_{i}^{(\alpha)}, \omega^{(\alpha)}, \theta^{(\alpha)}, \varepsilon_{i j}^{(\alpha)}, \mu_{i j}^{(\alpha)}, t_{i j}^{(\alpha)}, m_{i j}^{(\alpha)},\right. \\
\left.\lambda_{i}^{(\alpha)}, s^{(\alpha)}, q_{i}^{(\alpha)}\right\}, \quad \alpha=1,2 .
\end{gathered}
$$

For the sake of simplicity, we now introduce the notations

$$
\begin{aligned}
t_{i}=t_{i j} n_{j}, & T_{i}=t_{i j}^{+} v_{j}, \\
m_{i}=m_{i j} n_{j}, & M_{i}=m_{i j}^{+} v_{j}, \\
\lambda=\lambda_{i} n_{i}, & \Lambda=\lambda_{i}^{+} v_{i}, \\
q=q_{i} n_{i}, & Q=q_{i}^{+} v_{i} .
\end{aligned}
$$

In the following theorem, we prove a reciprocity relation of Betti type.

Theorem 2. If a microstretch thermoelastic body is subjected to two systems of loadings $\mathscr{G}^{(\alpha)}$ then between the corresponding solutions $\mathcal{S}^{(\alpha)}$ there is the following reciprocity relation:

$$
\begin{aligned}
& \int_{B}\left(f_{i}^{(1)} * u_{i}^{(2)}+g_{i}^{(1)} * \varphi_{i}^{(2)}+l^{(1)} * \omega^{(2)}\right. \\
& \left.-\frac{1}{T_{0}} g * w^{(1)} * \theta^{(2)}\right) d V \\
& +\int_{\partial B} g *\left(t_{i}^{(1)} * u_{i}^{(2)}+m_{i}^{(1)} * \varphi_{i}^{(2)}+\lambda^{(1)} * \omega^{(2)}\right. \\
& \left.-\frac{1}{T_{0}} h * q^{(1)} * \theta^{(2)}\right) d A \\
& -\int_{\Sigma} g *\left(T_{i}^{(1)} * U_{i}^{(2)}+M_{i}^{(1)} * \Phi_{i}^{(2)}+\Lambda^{(1)} * \Psi^{(2)}\right. \\
& \left.-\frac{1}{T_{0}} h * Q^{(1)} * \Theta^{(2)}\right) d A \\
& =\int_{B}\left(f_{i}^{(2)} * u_{i}^{(1)}+g_{i}^{(2)} * \varphi_{i}^{(1)}+l^{(2)} * \omega^{(1)}\right. \\
& \left.-\frac{1}{T_{0}} g * w^{(2)} * \theta^{(1)}\right) d V \\
& +\int_{\partial B} g *\left(t_{i}^{(2)} * u_{i}^{(1)}+m_{i}^{(2)} * \varphi_{i}^{(1)}+\lambda^{(2)} * \omega^{(1)}\right. \\
& \left.-\frac{1}{T_{0}} h * q^{(2)} * \theta^{(1)}\right) d A \\
& -\int_{\Sigma} g *\left(T_{i}^{(2)} * U_{i}^{(1)}+M_{i}^{(2)} * \Phi_{i}^{(1)}+\Lambda^{(2)} * \Psi^{(1)}\right. \\
& \left.-\frac{1}{T_{0}} h * Q^{(2)} * \Theta^{(1)}\right) d A .
\end{aligned}
$$

Proof. In view of symmetry relations (6) and with the aid of the constitutive relations (4), by direct calculations it is easy to obtain

$$
\begin{aligned}
& t_{i j}^{(1)} * \varepsilon_{i j}^{(2)}+m_{i j}^{(1)} * \mu_{i j}^{(2)}+\lambda_{i}^{(1)} * \gamma_{i}^{(2)}+s^{(1)} * \omega^{(2)} \\
&-\varrho \theta^{(1)} * \eta^{(2)}= t_{i j}^{(2)} * \varepsilon_{i j}^{(1)}+m_{i j}^{(2)} \\
& * \mu_{i j}^{(1)}+\lambda_{i}^{(2)} * \gamma_{i}^{(1)}+s^{(2)} \\
& * \omega^{(1)}-\varrho \theta^{(2)} * \eta^{(1)} .
\end{aligned}
$$

Let us introduce the notation

$$
\begin{gathered}
I_{\alpha \beta}=\int_{B} g *\left[t_{i j}^{(\alpha)} * \varepsilon_{i j}^{(\beta)}+m_{i j}^{(\alpha)} * \mu_{i j}^{(\beta)}+\lambda_{i}^{(\alpha)} * \gamma_{i}^{(\beta)}\right. \\
\left.+s^{(\alpha)} * \omega^{(\beta)}-\varrho \theta^{(\alpha)} * \eta^{(\beta)}\right] d V
\end{gathered}
$$

for $\alpha, \beta=1,2$

Based on identity (21) and notation (22), it is easy to see that

$$
I_{\alpha \beta}=I_{\beta \alpha} .
$$

With the aid of the equations of motion and (15), we can write

$$
\begin{aligned}
g *\left[t_{i j}^{(\alpha)} * \varepsilon_{i j}^{(\beta)}+m_{i j}^{(\alpha)} * \mu_{i j}^{(\beta)}+\lambda_{i}^{(\alpha)} * \gamma_{i}^{(\beta)}\right. \\
\left.\quad+s^{(\alpha)} * \omega^{(\beta)}-\varrho \theta^{(\alpha)} * \eta^{(\beta)}\right] \\
=g *\left[t_{j i}^{(\alpha)} * u_{j}^{(\beta)}+m_{j i}^{(\alpha)} * \varphi_{j}^{(\beta)}+\lambda_{i}^{(\alpha)} * \omega^{(\beta)}\right. \\
\left.\quad-\frac{1}{T_{0}} h * q_{i}^{(\alpha)} * \theta^{(\beta)}\right]_{, i} \\
+f_{i}^{(\alpha)} * u_{i}^{(\beta)}+g_{i}^{(\alpha)} * \varphi_{i}^{(\beta)}+l^{(\alpha)} * \omega^{(\beta)} \\
\quad-\frac{1}{T_{0}} g * w^{(\alpha)} * \theta^{(\beta)} \\
-\left[\varrho u_{i}^{(\alpha)} * u_{i}^{(\beta)}+I_{i j} \varphi_{i}^{(\alpha)} * \varphi_{j}^{(\beta)}+J \omega^{(\alpha)} * \omega^{(\beta)}\right] \\
+\frac{1}{T_{0}} g * h * k_{i j} \theta_{, i}^{(\alpha)} * \theta_{, j}^{(\beta)} .
\end{aligned}
$$

By integrating in (24) and using the divergence theorem, we are lead to

$$
\begin{gathered}
I_{\alpha \beta}=\int_{B}\left(f_{i}^{(\alpha)} * u_{i}^{(\beta)}+g_{i}^{(\alpha)} * \varphi_{i}^{(\beta)}+l^{(\alpha)} * \omega^{(\beta)}\right. \\
\left.-\frac{1}{T_{0}} g * w^{(\alpha)} * \theta^{(\beta)}\right) d V \\
+\int_{\partial B} g *\left(t_{i}^{(\alpha)} * u_{i}^{(\beta)}+m_{i}^{(\alpha)} * \varphi_{i}^{(\beta)}+\lambda^{(\alpha)} * \omega^{(\beta)}\right. \\
\left.-\frac{1}{T_{0}} h * q^{(\alpha)} * \theta^{(\beta)}\right) d A
\end{gathered}
$$




$$
\begin{gathered}
-\int_{\Sigma} g *\left(T_{i}^{(\alpha)} * U_{i}^{(\beta)}+M_{i}^{(\alpha)} * \Phi_{i}^{(\beta)}+\Lambda^{(\alpha)} * \Psi^{(\beta)}\right. \\
\left.-\frac{1}{T_{0}} h * Q^{(\alpha)} * \Theta^{(\beta)}\right) d A
\end{gathered}
$$

Finally, introducing (25) into (22), we arrive at the desired result (20).

It is easy to see that in the absence of the discontinuities we obtain the generalization, in the context of the thermoelasticity of thermoelastic microstretch bodies, of the previous results established in the classical thermoelastodynamics.

Based on relation (20), we now calculate the thermomechanical body loadings equivalent to a given dislocation. To this aim, we assume that $u_{i}^{(2)}, \varphi_{i}^{(2)}, \omega^{(2)}$, and $\theta^{(2)}$, as functions of $(t, x)$, where $x=\left(x_{i}\right)$, are of class $C^{\infty}(B \times[0, \infty))$. Of course, if the functions $u_{i}^{(2)}, \varphi_{i}^{(2)}, \omega^{(2)}$, and $\theta^{(2)}$ are given, then by means of (15), we can determine the functions $F_{i}^{(2)}, G_{i}^{(2)}$, $L^{(2)}$, and $r^{(2)}$.

Now, we restrict our considerations only to the case when $U_{i}^{(2)}=\Phi_{i}^{(2)}=0, i=1,2,3, \Psi^{(2)}=\Theta^{(2)}=0$, and $\eta^{(2)}$ correspond to the faulted medium. Then, in the case of identical boundary conditions, we obtain

$$
\begin{gathered}
\int_{B} \varrho\left(F_{i}^{(1)} * u_{i}^{(2)}+G_{i}^{(1)} * \varphi_{i}^{(2)}+L^{(1)} * \omega^{(2)}\right. \\
\left.-\frac{1}{T_{0}} h * r^{(1)} * \theta^{(2)}\right) d V \\
=\int_{B} \varrho\left(F_{i}^{(2)} * u_{i}^{(1)}+G_{i}^{(2)} * \varphi_{i}^{(1)}+L^{(2)} * \omega^{(1)}\right. \\
\left.-\frac{1}{T_{0}} h * r^{(2)} * \theta^{(1)}\right) d V \\
-\int_{\Sigma} g *\left(T_{i}^{(2)} * U_{i}^{(1)}+M_{i}^{(2)} * \Phi_{i}^{(1)}+\Lambda^{(2)} * \Psi^{(1)}\right. \\
\left.-\frac{1}{T_{0}} h * Q^{(2)} * \Theta^{(1)}\right) d A .
\end{gathered}
$$

In view of (19), we have

$$
\begin{gathered}
T_{i}^{(2)}=\left[A_{i j m n} \varepsilon_{m n}+B_{i j m n} \mu_{m n}+D_{i j m} \gamma_{m}+a_{i j} \omega-E_{i j} \theta\right] \nu_{j}, \\
M_{i}^{(2)}=\left[B_{i j m n} \varepsilon_{m n}+C_{i j m n} \mu_{m n}+E_{i j m} \gamma_{m}+b_{i j} \omega-D_{i j} \theta\right] \nu_{j}, \\
\Lambda^{(2)}=\left[D_{m n i} \varepsilon_{m n}+E_{m n i} \mu_{m n}+C_{i j} \gamma_{j}+d_{i} \omega-L_{i} \theta\right] \nu_{i}, \\
\Theta^{(2)}=k_{i j} \theta_{, i} \nu_{j} .
\end{gathered}
$$

Taking into account the definition of the Dirac translated measure, $\delta$, we can prove the relation of the following type:

$$
\begin{aligned}
& \psi_{i}(\xi, t)=\int_{B} \psi_{i}(x, t) \delta(x-\xi) d V, \\
& \psi_{i, j}(\xi, t)=\int_{B} \psi_{i}(x, t) \delta_{, j}(x-\xi) d V
\end{aligned}
$$

and then the relation (26) can be rewritten as follows:

$$
\begin{aligned}
& \int_{B} \varrho\left[\left(F_{i}^{(1)}+\mathscr{F}_{i}\right) * u_{i}^{(2)}+\left(G_{i}^{(1)}+\mathscr{G}_{i}\right) * \varphi_{i}^{(2)}\right. \\
& \left.\quad+\left(L^{(1)}+\mathscr{L}\right) * \omega^{(2)}-\frac{1}{T_{0}} *\left(r^{(1)}+\mathscr{R}\right) * \theta^{(2)}\right] d V \\
& =\int_{B} \varrho\left(F_{i}^{(2)} * u_{i}^{(1)}+G_{i}^{(2)} * \varphi_{i}^{(1)}+L^{(2)} * \omega^{(1)}\right. \\
& \left.-\frac{1}{T_{0}} h * r^{(2)} * \theta^{(1)}\right) d V .
\end{aligned}
$$

In the above relations we have used the notations

$$
\begin{gathered}
\mathscr{F}_{k}=-\frac{1}{\varrho} \int_{\Sigma}\left[A_{j i r k} U_{i}^{(1)}+B_{j i r k} \Phi_{i}^{(1)}+D_{j k r} \Psi^{(1)}\right] \\
\cdot \delta_{, r}(x-\xi) v_{j} d A_{\xi}, \\
\mathscr{G}_{k}=-\frac{1}{\varrho} \int_{\Sigma}\left[\left(B_{j i r k} U_{i}^{(1)}+C_{j i r k} \Phi_{i}^{(1)}+E_{j k r} \Psi^{(1)}\right) \delta_{, r}(x-\xi)\right. \\
+\varepsilon_{m n k}\left(A_{j i m n} U_{i}^{(1)}+B_{j i m n} \Phi_{i}^{(1)}+D_{j m n} \Psi^{(1)}\right) \\
\cdot \delta(x-\xi)] v_{j} d A_{\xi}, \\
\mathscr{L}=-\frac{1}{\varrho} \int_{\Sigma}\left[\left(D_{j i m} U_{i}^{(1)}+E_{j i m} \Phi_{i}^{(1)}+C_{j m} \Psi^{(1)}\right) \delta_{, m}\right. \\
\cdot(x-\xi)+\left(a_{j i} U_{i}^{(1)}+b_{j i} \Phi_{i}^{(1)}+d_{j} \Psi^{(1)}\right) \\
\cdot \delta(x-\xi)] v_{j} d A_{\xi}, \\
\mathscr{R}=\frac{1}{\varrho} \int_{\Sigma} T_{0}\left[\left(E_{j i} U_{i}^{(1)}+D_{j i} \Phi_{i}^{(1)}+L_{j} \Psi^{(1)}\right) \delta(x-\xi)\right. \\
\left.-k_{i j} \delta_{, i}(x-\xi)\right] v_{j} d A_{\xi} \cdot
\end{gathered}
$$

In order to illustrate theoretical results derived in the previous sections, we present some numerical results taking into account the initial conditions given in relations (10).

Figure 1 shows the $3 \mathrm{D}$ variation of displacement with respect to time and space. Because we are interested only in the positive values of time, it is observed that the displacement increases sharply with $x$. The 3D variation of microstretch function with respect to time and space is shown in Figure 2. It is observed that for an increasing of time the microstretch function tends to become constant and the microstretch surface becomes a parallel plane with $x O t$. 


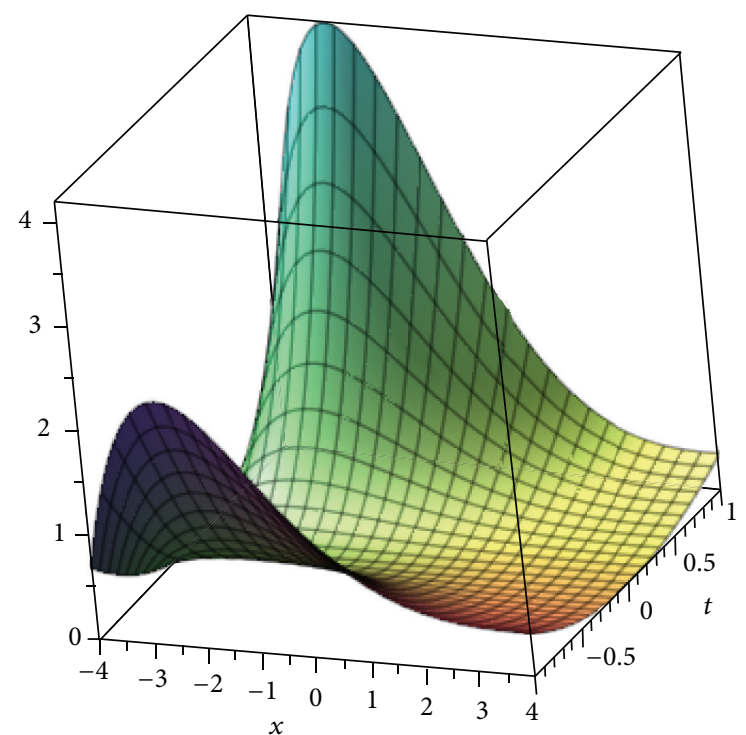

Figure 1: The variation of $u(x, t)$.

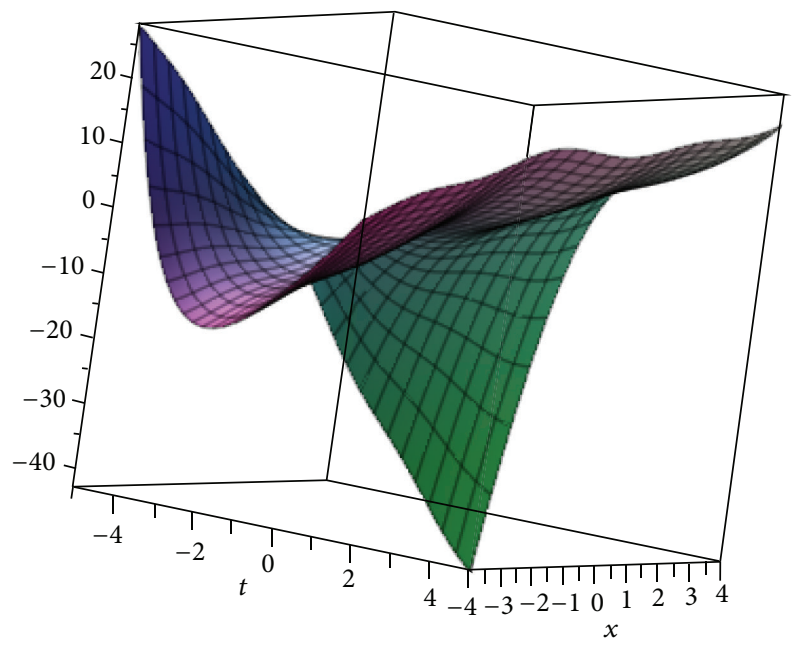

FIgURE 2: The variation of $\omega(x, t)$.

In Figure 3 the 3D variation of microrotation function is represented and Figure 4 shows the variation of the temperature with increasing $x$ under the effects of thermoelastic body.

Figure 5 presents the displacement profiles $u(x, t)$ against $x$ at different times. The displacement achieves a maximum for all chosen times for $x \in(-2.5 ;-2.35)$ The displacement is an increasing function with respect to space fot $x \in$ $[-5 ;-2.5] \cup[3.4 ; 6.3]$, it is a decreasing function with respect to space for $x \in(-2.5 ; 3.4)$ and tends to a steady-state.

Figure 6 presents the microstretch profiles $\omega(x, t)$ against $x$ at different times. The microstretch is an increasing function with respect to time. It is clear that the microstretch function is not stable.

The profiles of microrotation function $\phi(x, t)$ over space are shown in Figure 7 for four values of time. The microrotation function, as it results from Figure 4, is a decreasing function with respect to time.

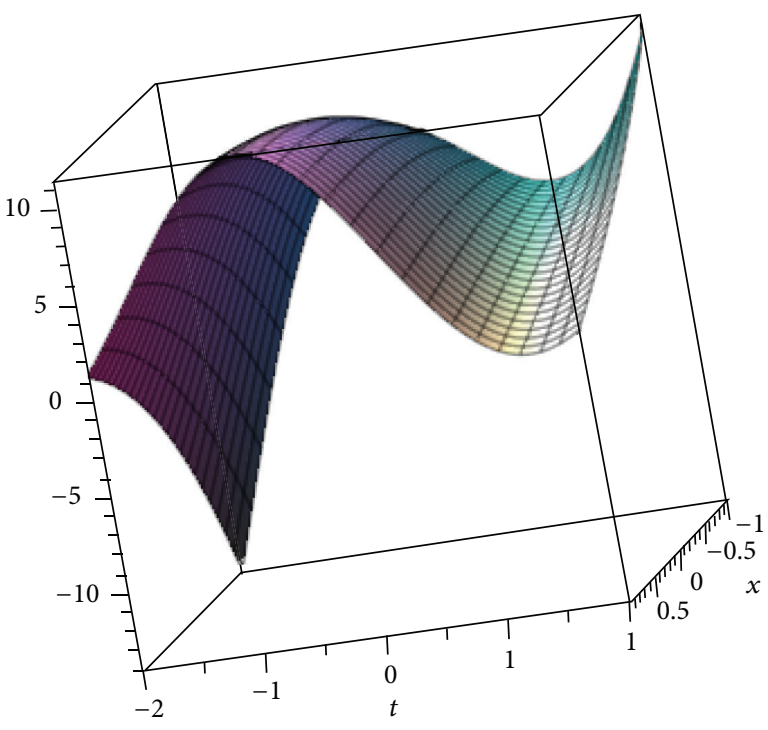

Figure 3: The variation of $\phi(x, t)$.

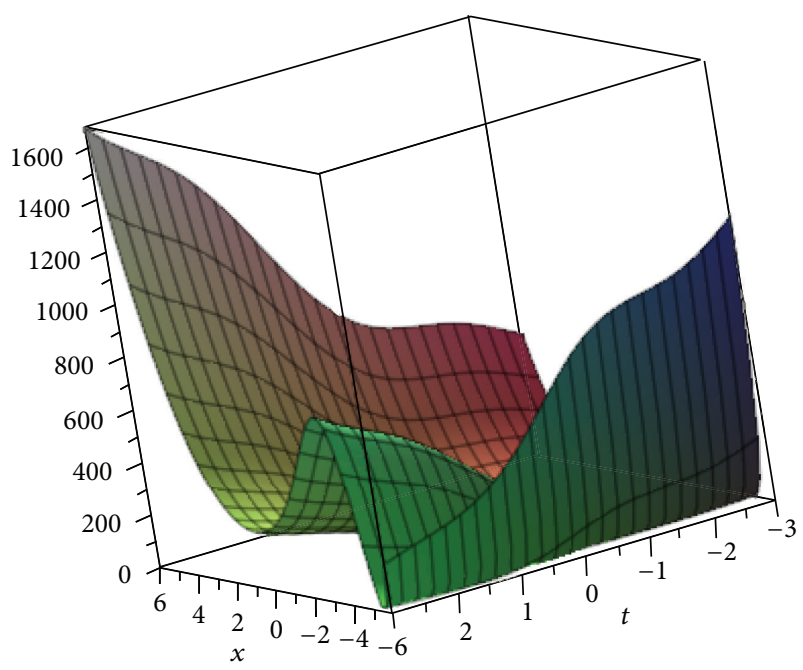

FIGURE 4: The variation of $\theta(x, t)$.

Figure 8 presents the temperature profiles $\theta(x, t)$ against $x$ at different times. The medium temperature is an increasing function with respect to time for $x>0$.

Based on papers by Eringen [1,14] and Kumar et al. [12] and based on the simulations performed in the laboratory of virtual engineering, we have used the following characteristic constitutive coefficients for the microstretch thermoelastic materials (in the isotropic case):

$$
\begin{array}{cc}
\lambda=9.4 \cdot 10^{10} \mathrm{Nm}^{-2}, & \mu=4 \cdot 10^{10} \mathrm{NM}^{-2}, \\
\rho=1.74 \cdot 10^{3} \mathrm{Kgm}^{-3}, & \kappa=10^{10} \mathrm{Nm}^{-2}, \\
\alpha=0.779 \cdot 10^{-9} \mathrm{~N}, & \eta=1.22 \mathrm{~kJ} / \mathrm{mol}, \\
a_{1}=0.779 \cdot 10^{-9} \mathrm{~N}, & a_{2}=0.19 \cdot 10^{-19}
\end{array}
$$




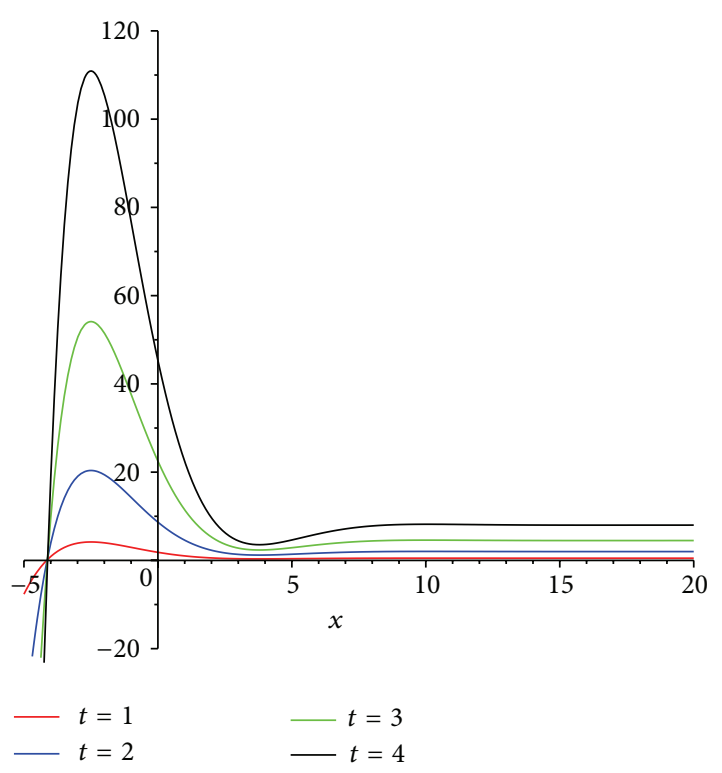

Figure 5: The behavior of $u(x, t)$ at different values of time.

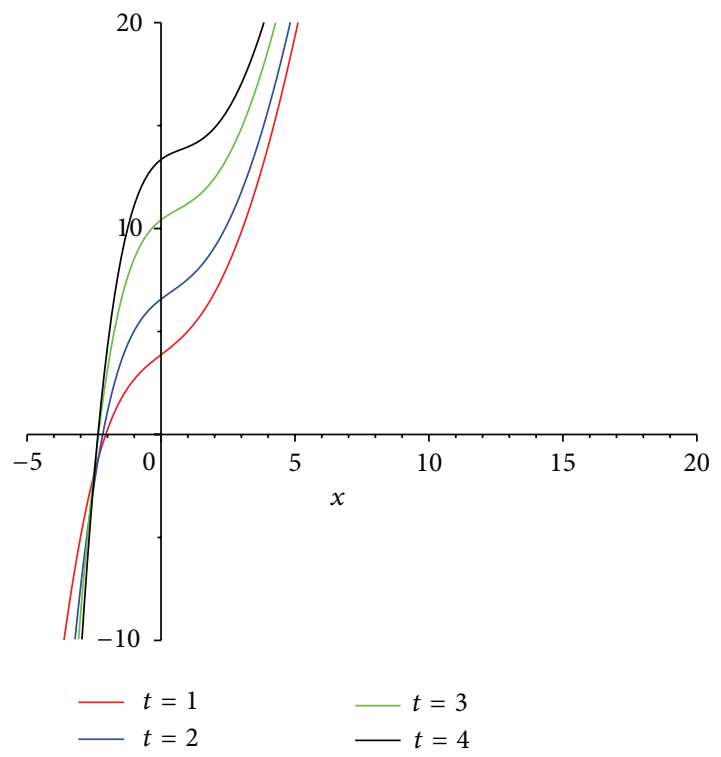

FIgURE 6: The behavior of $\omega(x, t)$ at different values of time.

$$
\begin{array}{cc}
b_{1}=0.72 \cdot 10^{-17}, & c_{1}=0.227 \cdot 10^{-11}, \\
d_{1}=0.723 \cdot 10^{-19}, & e_{1}=0.315 \cdot 10^{-17}, \\
a_{3}=1.7 \cdot 10^{-4}, & b_{2}=1.26 \cdot 10^{-6}, \\
c_{2}=0.97 \cdot 10^{-5} ; & c_{3}=0.779 \cdot 10^{-9} ; \\
e_{2}=1.48 \cdot 10^{-5} ; & d_{2}=0.84 \cdot 10^{-3}, \\
l_{1}=0.64 \cdot 10^{-7} .
\end{array}
$$

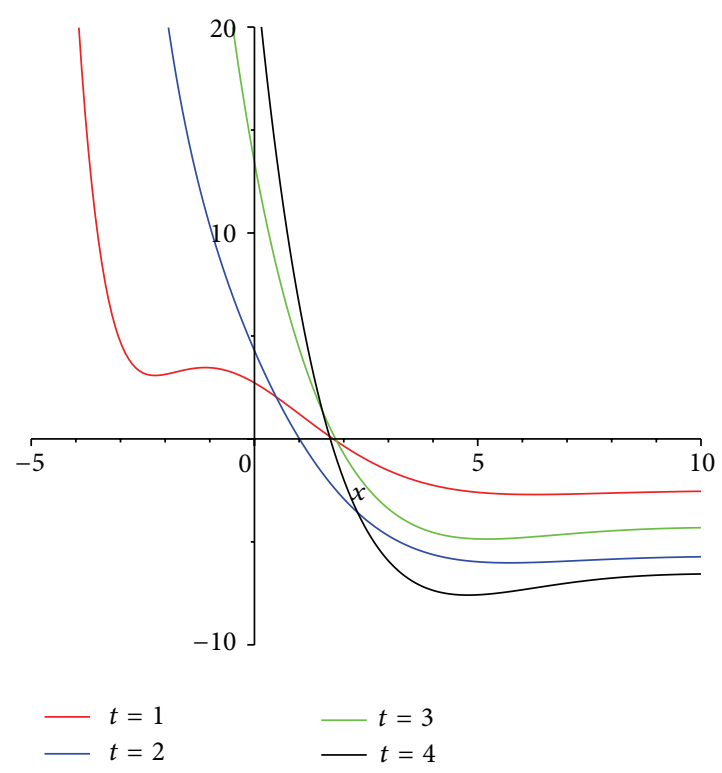

Figure 7: The behavior of $\phi(x, t)$ at different values of time.

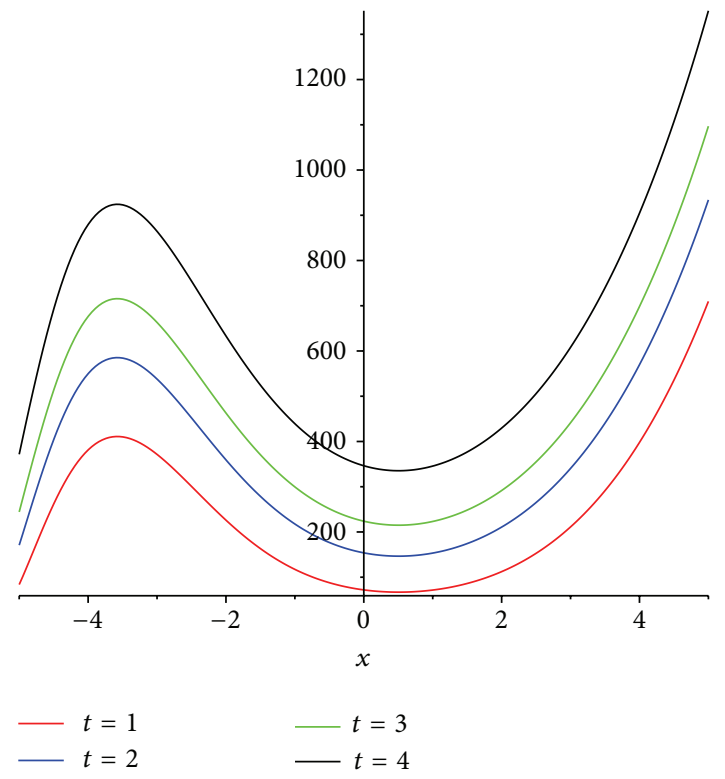

FIGURE 8: The behavior of $\theta(x, t)$ at different values of time.

It is observed that for the displacement vector greater values than $x=3.2$ the solution is stabilized. The microstretch function increases asymptotically to $x=5$. The microrotation vector is stabilized for all chosen moment starting with $x$ in the range $(3.6,3.85)$. The temperature increases for $x \in$ $(-5,-3.5)$, decreases for $x \in(-3.5,0)$, and then increases asymptotically to $x=5$.

\section{Conclusions}

In the absence of the discontinuities we obtain the generalization, in the context of the thermoelasticity of thermoelastic 
microstretch bodies, of the previous results established in the classical thermoelastodynamics.

In the classical elasticity in the system of equations of motion occur only three unknown functions, while in our case the number of unknowns is seven.

Also, while in the classical elasticity the equations of motion are parabolic, hyperbolic, or elliptic only, in our case we have a mixed system of equations.

Taking into account relation (29) we deduce that the effect of the discontinuities across the surface $\Sigma$ can be represented by extra external body loads and heat supply.

Although these are supposed to act in an unfaulted medium and cannot in any sense represent real forces acting in the real medium, they may nevertheless provide, as pointed in $[3,4,7]$, a useful theoretical tool and this because if two dislocations have the same equivalent force, they also emit the same radiation.

\section{Conflict of Interests}

The authors declare that there is no conflict of interests regarding the publication of this paper.

\section{Acknowledgments}

(1) The work of the second author of this paper is supported by the Sectoral Operational Programme Human Resources Development (SOP HRD), financed from the European Social Fund and by the Romanian Government under the Project no. POSDRU/159/1.5/S/134378. (2) The authors are grateful to the reviewers for their valuable suggestions and comments which improve the value of the paper.

\section{References}

[1] A. C. Eringen, "Theory of thermomicrostretch elastic solids," International Journal of Engineering Science, vol. 28, no. 12, pp. 1291-1301, 1990.

[2] E. Boschi and F. Mainardi, "Body loadings in thermomicrostretch elastic solids," Geophysical Journal of the Royal Astronomical Society, vol. 34, pp. 313-320, 1973.

[3] A. T. De Hoop, "A domain of influence in elasticity," Applied Scientific Research, vol. 16, pp. 39-45, 1966.

[4] A. T. De Hoop, Handbook of Radiation, Academic Press, New York, NY, USA, 1995.

[5] D. Ieşan, "Some applications of micropolar mechanics to earthquake problems," International Journal of Engineering Science, vol. 19 , no. 6, pp. 855-864, 1981.

[6] D. Ieşan and R. Quintanilla, "Some theorems in the theory of microstretch thermopiezoelectricity," International Journal of Engineering Science, vol. 45, no. 1, pp. 1-16, 2007.

[7] A. K. Chatterjee and L. Knopoff, "Crack breakout dynamics," Bulletin Seismological Society of America, vol. 80, no. 6, pp. 15711579, 1990.

[8] L. Knopoff, J. A. Landoni, and M. S. Abinante, "Dynamical model of an earthquake fault with localization," Physical Review $A$, vol. 46, no. 12 , pp. 7445-7449, 1992.

[9] M. I. Marin, R. P. Agarwal, and S. R. Mahmoud, "Nonsimple material problems addressed by the Lagrange's identity," Boundary Value Problems, vol. 2013, article 135, 2013.
[10] M. Marin and G. Stan, "Weak solutions in elasticity of dipolar bodies with stretch," Carpathian Journal of Mathematics, vol. 29, no. 1, pp. 33-40, 2013.

[11] M. Marin and O. Florea, "On temporal behaviour of solutions in thermoelasticity of porous micropolar bodies," Analele Stiintifice ale Universitatii Ovidius Constanta, vol. 22, no. 1, pp. 169188, 2014.

[12] R. Kumar, S. Ahuja, and S. K. Garg, "Rayleigh waves in isotropic microstretch thermoelastic diffusion solid half space," Latin American Journal of Solids and Structures, vol. 11, no. 2, pp. 299319, 2014.

[13] K. Sharma and M. Marin, "Reflection and transmission of waves from imperfectboundary between two heat conducting micropolarthermoelastic solids," Analele Stiintifice ale Universitatii Ovidius Constanta, vol. 22, no. 2, pp. 151-175, 2014.

[14] A. C. Eringen, Microcontinuum Field Theories. I. Foundations and solids, Springer, New York, NY, USA, 1999. 


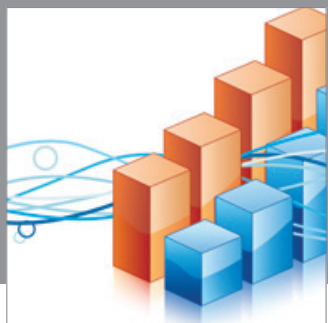

Advances in

Operations Research

mansans

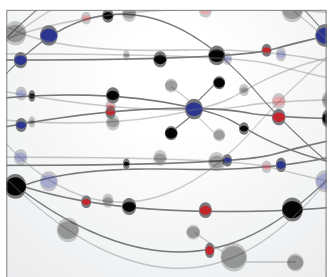

The Scientific World Journal
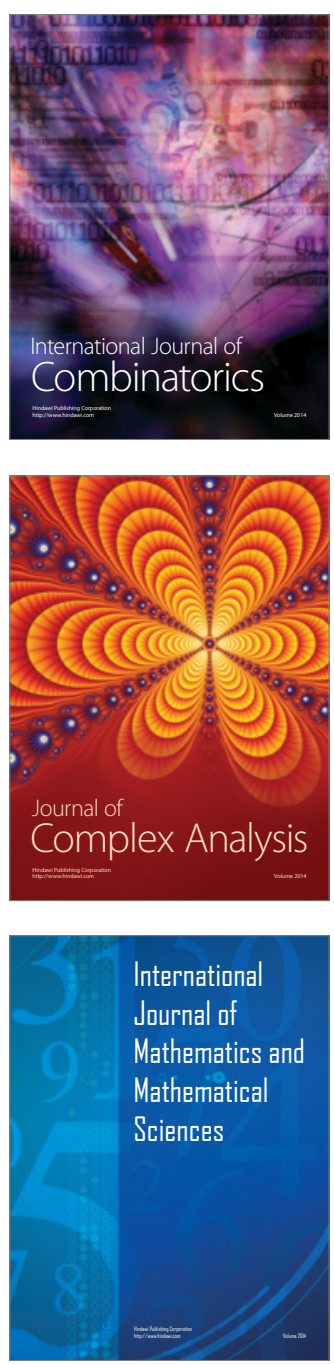
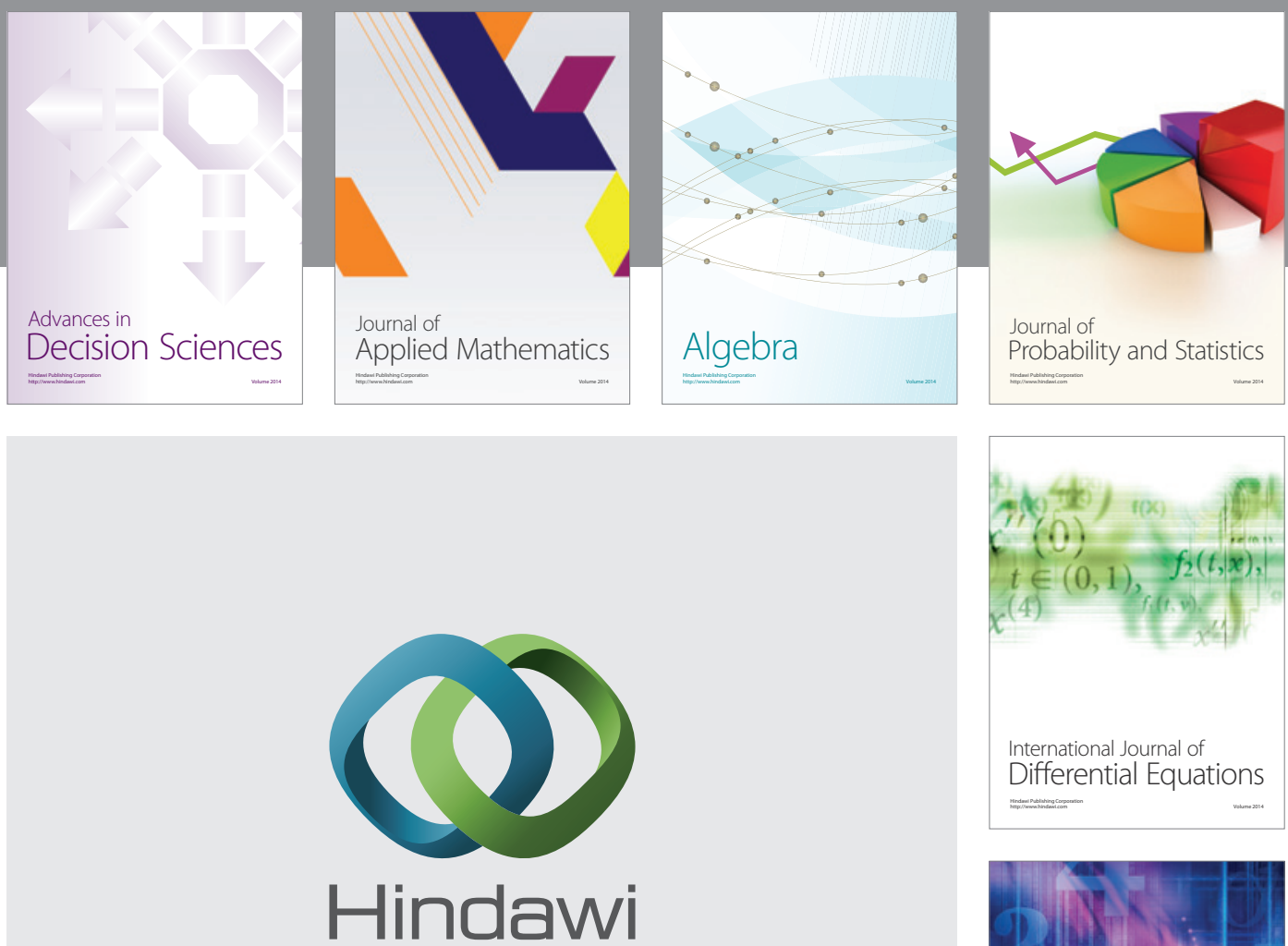

Submit your manuscripts at http://www.hindawi.com
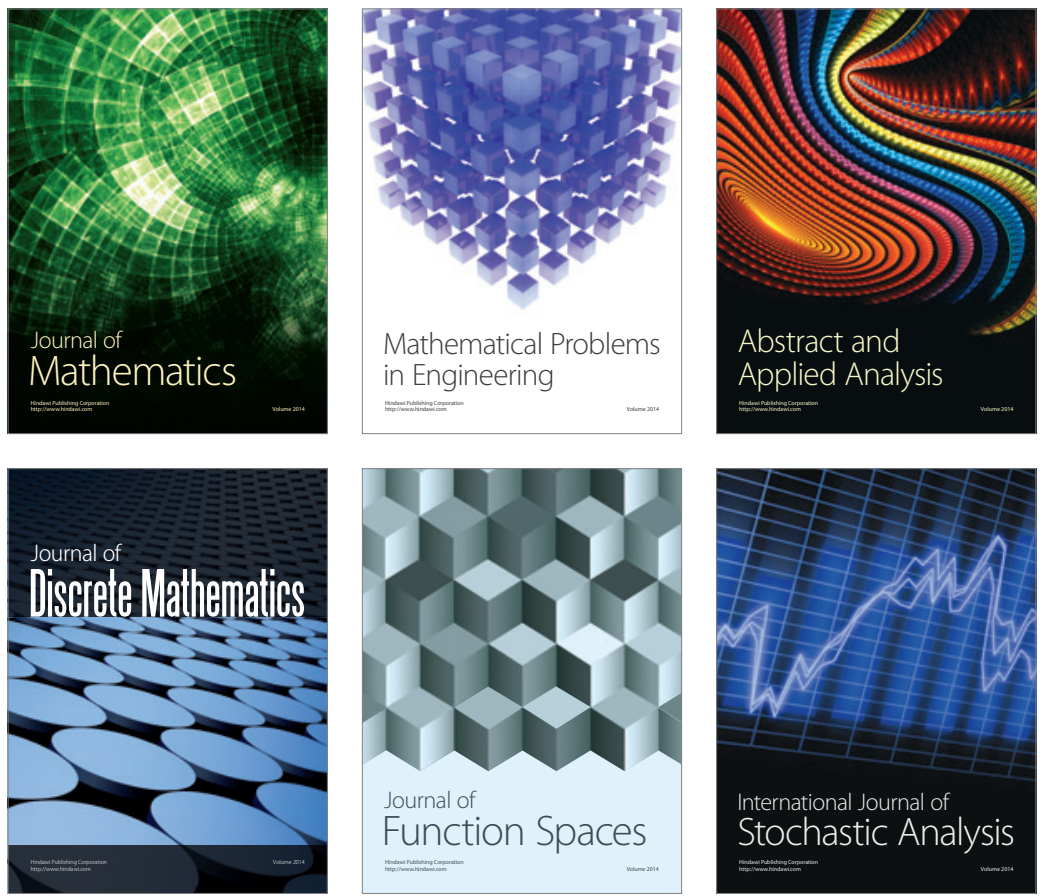

Journal of

Function Spaces

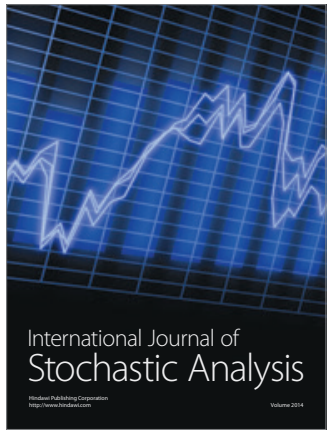

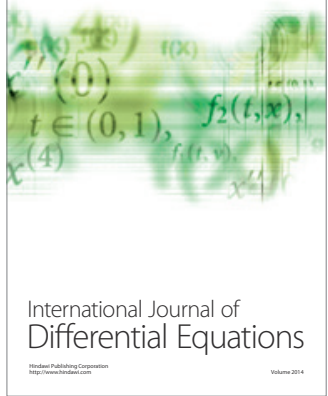
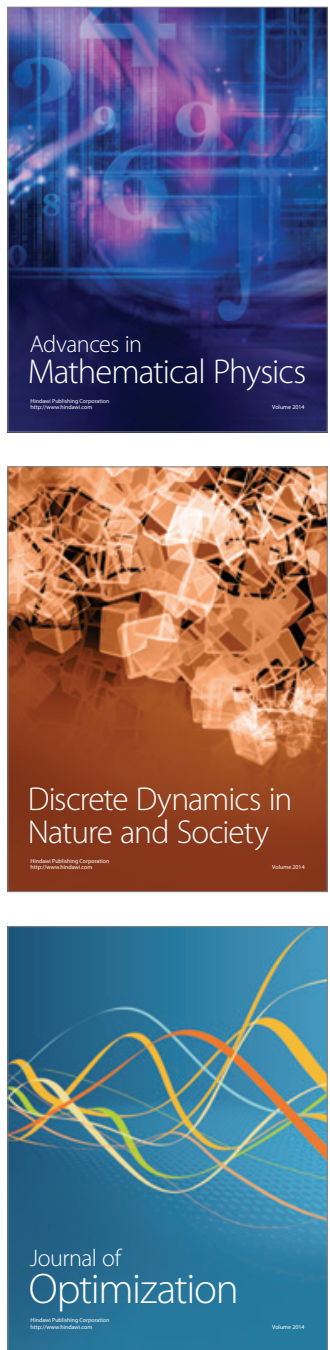